\title{
Huge Retroperitoneal Hematoma Following Oblique Lumbar Interbody Fusion
}

\author{
Jong Yang $\mathrm{Oh}^{1}$, Ji-Hoon Seong ${ }^{2}$, Jin-Sung Kim², Choon Keun Park ${ }^{3}$ \\ ${ }^{1}$ Department of Neurosurgery, Spine Center, Saeng Saeng Hospital, Bucheon, \\ ${ }^{2}$ Department of Neurosurgery, Spine Center, Seoul St. Mary's Hospital, The Catholic University of Korea College of Medicine, Seoul, \\ ${ }^{3}$ Department of Neurosurgery, Spine Center, Suwon Wiltse Memorial Hospital, Suwon, Korea
}

Corresponding Author:

Jin-Sung Kim, MD, PhD

Professor, Department of Neurosurgery, Spine Center, Seoul St. Mary's Hospital,

The Catholic University of Korea College of Medicine, 222 Banpo-daero, Seocho-gu, Seoul 06591, Korea

Tel: $+82-2-2258-6128$

Fax: +82-2-594-4248

E-mail: mddavidk@gmail.com; mdlukekim@gmail.com

Received: February 15, 2017

Revised: May 23, 2017

Accepted: May 24, 2017

\begin{abstract}
A 64-year-old man who had lumbar spinal stenosis underwent oblique lumbar interbody fusion (OLIF) with cortical screw fixation at the L2-3, L3-4, and L4-5 levels. During the operation, there was no event of serious surgical bleeding. After the operation, he complained of severe flank pain and back pain. A computerized tomography (CT) scan identified a huge amount of retroperitoneal hematoma compressing peritoneum and the patient underwent exploration immediately. There was active arterial bleeding at the drain puncture site. The active arterial bleeding was controlled and the retroperitoneal hematoma was removed. The patient's symptoms were recovered after the second operation. A huge amount of retroperitoneal hematoma after OLIF requiring reoperation is rare. Meticulous bleeding control and repeated inspection of the drain puncture site are critical prior to wound closure. In addition, for patients presenting with severe pain after the operation, rapid evaluation using $\mathrm{CT}$ and second operation is required as soon as possible.
\end{abstract}

Key Words: Oblique lumbar interbody fusion, Retroperitoneal hematoma, Postoperative bleeding, Reoperation

\section{INTRODUCTION}

Minimally invasive oblique lumbar interbody fusion (OLIF) is being increasingly utilized as an alternative to conventional anterior or posterior procedures ${ }^{3,10)}$. This procedure has several advantages, including minimal surgical trauma, low intraoperative blood loss, decreased incisional discomfort, postoperative morbidity, and improved recovery time ${ }^{6)}$. Among the complications, despite minimal blood loss, is the possibility of postoperative huge amounts of retroperitoneal hematoma, which then requires a second operation, although this is extremely rare. The authors report a case of a huge amount of retroperitoneal hematoma after OLIF and consider its causes.

\section{CASE REPORT}

A 64-year-old man presented with lower back pain, pain in both legs and paresthesia radiating from the buttocks to the toe tip, persisting for 3 years. Non-operative supportive treatment, including medication, physiotherapy, and epidural steroid injection, could not relieve the patient's symptom. The patient could not walk for 5 minutes because of severe pain in both legs and neurogenic claudication. Upon neurogenic examination, both ankles dorsiflexion and great toe dorsiflexion were grade IV and paresthesia and hypoesthesia were observed on the $\mathrm{L} 5$ dermatome. A plain radiograph showed retrolisthesis at L2-3 and L3-4, and spondylolisthesis at L4-5 (Fig. 1). MRI

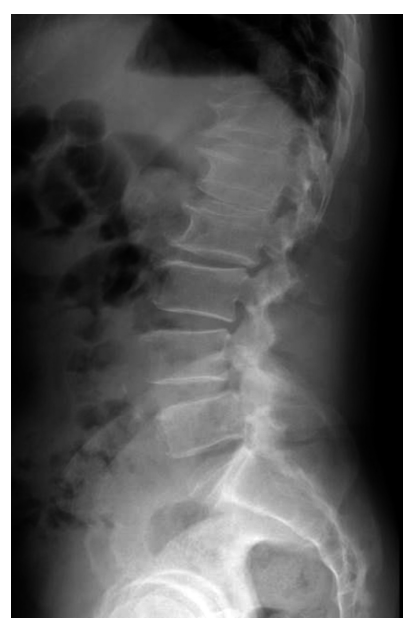

Fig. 1. Plain radiograph showing retrolisthesis at $\mathrm{L} 2-3$ and L3-4 levels, and spondylolisthesis at L4-5 level. 


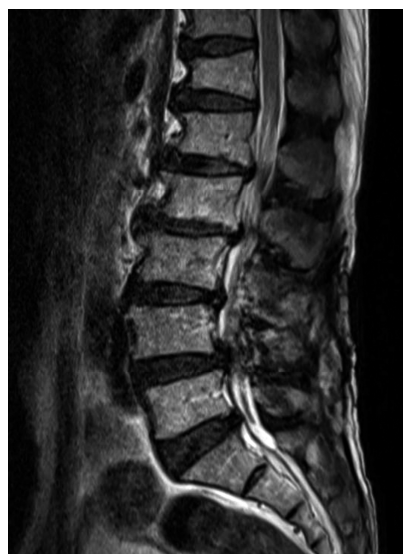

Fig. 2. Sagittal magnetic resonance image showing disc herniation and severe central stenosis at L2-3, L3-4 and L4-5 levels.
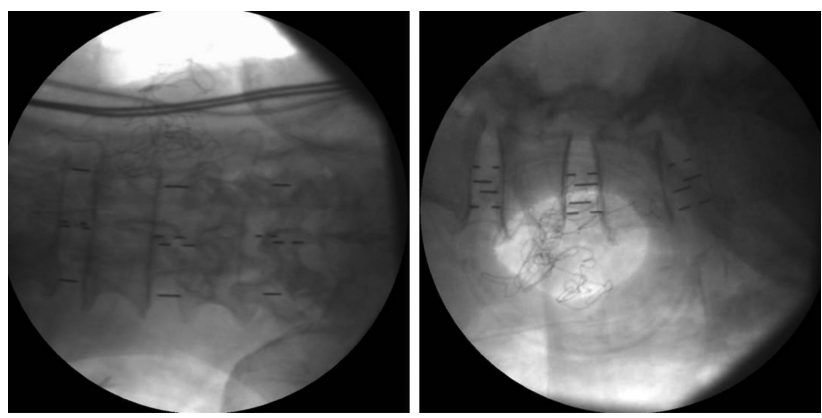

Fig. 3. Intraoperative $\mathrm{C}$-arm fluoroscopic image showing well inserted cage at L2-3, L3-4 and L4-5 levels.
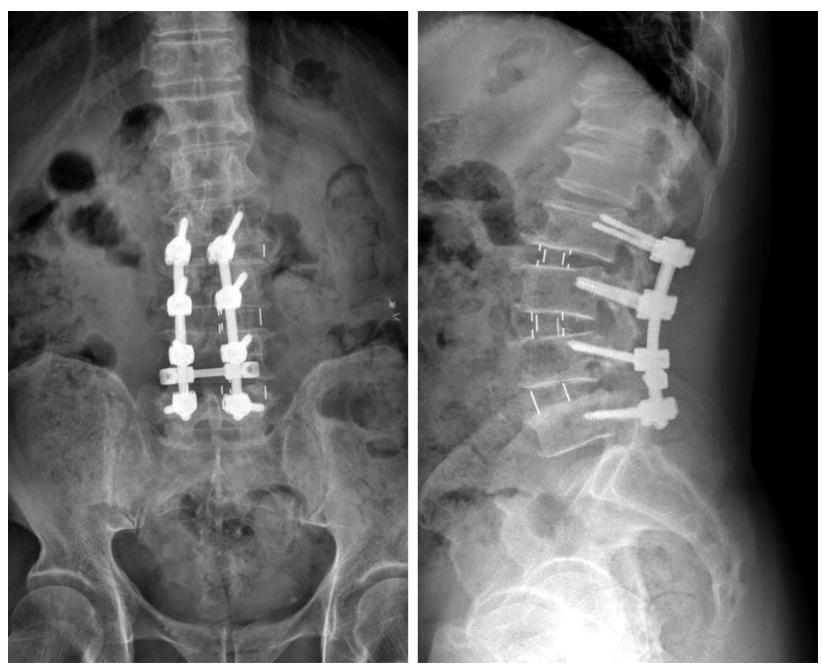

Fig. 4. Postoperative plain radiography showing successful insertion of the cage and cortical screw.

showed disc herniation and severe central stenosis at L2-3, L3-4, and L4-5 (Fig. 2). He underwent OLIF with a cortical screw fixation at L2-3, L3-4, and L4-5. At operation, we confirmed the insertion of a cage successfully using C-arm fluoroscopy (Fig. 3). There was no serious bleeding. After the operation, he complained of severe operation site pain, and flank pain
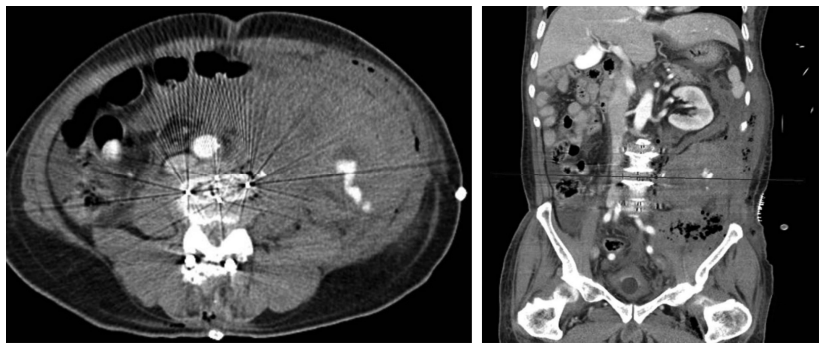

Fig. 5. Computerized tomography showing the huge amount retroperitoneal hematoma.

with large amounts of bleeding from the drainage bag. Postoperative plain radiography showed successful insertion of cage and cortical screw (Fig. 4). However, an immediate CT scan showed a huge amount of retroperitoneal hematoma compressing the peritoneum (Fig. 5). He underwent exploration. There was active arterial bleeding at the drain puncture site. Active arterial bleeding was controlled and a huge amount of retroperitoneal hematoma was removed. Meticulous hemostasis was performed, and we were assured that there was no further bleeding. We closed the operation wound in the usual manner with careful drain insertion. The patient's symptoms were improved after hematoma evacuation.

\section{DISCUSSION}

OLIF is a minimally invasive anterior lumbar interbody fusion (ALIF) that has been widely used in recent years. Its surgical technique was first introduced by Mayer with a microsurgical modification of the standard anterior approach to the lumbar spine in 19976). After that, Silvestre first mentioned the name OLIF when he published complications and morbidities of mini-open anterior retroperitoneal lumbar interbody fusion with Mayer's surgical technique ${ }^{10)}$.

OLIF is conducted by a $3-4 \mathrm{~cm}$ skin incision along the external oblique muscle in the lateral decubitus position. Using a blunt dissection, the psoas muscle is identified as an anatomical landmark. Disc space should be identified between the peritoneum and psoas muscle. An approximately $1 \mathrm{~cm}$-sized annulotomy is required to perform the discectomy and insert the autografts or allografts (e.g., the fusion cage). Such a minimally invasive approach may reduce operation time and the amount of bleeding $^{6)}$. Also, compared to ALIF, OLIF has several potential advantages, such as reduced injury to the abdominal musculature, and a reduced possibility of abdominal muscle atony and herniation of the abdominal wall ${ }^{2,5,7,9)}$.

For ALIF, there are a number of reports regarding complications. However, there are not many studies concerning the complication incidence of OLIF. Some known complications include vascular laceration, dural tear, sympathetic chain injury, neurologic deficiency, peritoneal laceration, transient ileus, incisional pain and retroperitoneal hematoma ${ }^{3,6,10)}$. As in this case, reports on the rapid progression of retroperitoneal hematoma after OLIF are extremely rare. Silvestre reported no retroperito- 
neal hematoma in their study of complications and morbidity after OLIF in 179 cases $^{10)}$, and Fujibayashi reported no retroperitoneal hematoma in their publication of results of OLIF across 52 levels $^{3)}$.

Kaiser reported retroperitoneal hematoma in the therapeutic result of mini-open retroperitoneal ALIF, but it was treated with conservative care ${ }^{5}$.

The known causes of retroperitoneal hematoma include ruptured aortic aneurysm, traumatic vascular injury, retroperitoneal neoplasm, and coagulopathy ${ }^{1)}$. Retroperitoneal hematoma after OLIF is an alternative etiology. This is a relatively rare but serious complication of OLIF. The common symptoms of retroperitoneal hematoma include abdominal pain, flank pain, groin pain, back pain, and hemodynamic instability ${ }^{8,11)}$. Rarely, it can be the cause of compressive femoral nerve palsy ${ }^{4)}$. Sunga reported that the spontaneous retroperitoneal hematomas mortality rate was 5.6\% within 7 days, $10.1 \%$ within 30 days, and $19.1 \%$ within 6 months ${ }^{11)}$. For traumatic retroperitoneal hematoma, the mortality rate was raised to $18-60 \%{ }^{12)}$. Retroperitoneal hematoma after OLIF is extremely rare, and there are no studies on its prognosis. For retroperitoneal hematoma occurring after retroperitoneal spine surgery, most of them are in small amount, and asymptomatic cases are more frequent than symptomatic cases.

In addition, most cases can be treated by conservative management, it is thought that reports of it are underestimated.

Many spine surgeons consider vascular injury that could not be discovered during procedure, bone bleeding at the fusion site, and muscle bleeding, as the cause of retroperitoneal hematoma. Therefore, close attention is paid to major vascular injury occurring during the operation. Complete hemostasis is attempted using bipolar electrocoagulation, monopolar cautery, bone wax, thrombin solution mixed gelfoam ${ }^{\circledR}$ (Pharmacia and Upjohn, Brigerwater, NJ, US), surgicel, and other hemostatic agents. Bone bleeding and muscle bleeding occur only at low levels and do not progress rapidly, while most are controlled because the veins are under low pressure. Although it is thought that postoperative retroperitoneal hematoma can be prevented by inserting a drain, this remains controversial. Despite such efforts, there may be bleeding that could not be discovered at the drain site, as in this case.

In this case, active arterial bleeding was observed at the drain puncture site which was inserted after the operation. High-pressured arterial bleeding can't be controlled, and it causes hypovolemic shock. Therefore, adequate management strategies are required according to the patient's symptoms. The drain puncture site compresses the periphery due to the drain and so bleeding may not even be identified. To prevent bleeding, when inserting the drain, the insertion site must be examined closely, and by moving the drain forward and backward to identify whether there is arterial bleeding. In addition, the location of drain puncture site must be considered carefully. There are many arteries supplying anterolateral abdominal wall muscles. $10^{\text {th }}, 11^{\text {th }}$ intercostal arteries, subcostal artery and ascending branch of a deep circumflex iliac artery are deep abdominal wall arteries and ascending branches of a superficial circumflex iliac artery and the superficial epigastric artery are superficial abdominal wall arteries. Among these abdominal wall arteries, $10^{\text {th }}, 11^{\text {th }}$ intercostal arteries and subcostal artery are relatively large arteries and located on relatively upper part of the abdominal wall than the others. Therefore, the lower part of an anterolateral abdominal wall is thought to be safe to insert the drain than the upper part. Also, there are many cases without symptoms, and so it is essential to carefully observe in the recovery room after the operation.

\section{CONCLUSION}

Rapid progression retroperitoneal hematoma occurring after OLIF is extremely rare. Active bleeding at the drain puncture site may aggravate hemodynamic instability. Therefore, it is necessary to make minute observation and hemostasis while inserting the drain and before suture, and even after the operation. A prompt second look operation should be conducted if there is pain and hemodynamic instability.

\section{REFERENCES}

1. Al-Khulaiwi A, Razaak FA, El Shair A, Bamehriz F: Idiopathic retroperitoneal hematoma. Ann Saudi Med 20:270-271, 2000

2. Eck JC, Hodges S, Humphreys SC: Minimally invasive lumbar spinal fusion. J Am Acad Orthop Surg 15:321-329, 2007

3. Fujibayashi S, Hynes RA, Otsuki B, Kimura H, Takemoto M, Matsuda S: Effect of indirect neural decompression through oblique lateral interbody fusion for degenerative lumbar disease. Spine (Phila Pa 1976) 40:E175-182

4. Gurbuz O, Ercan A, Kumtepe G, Karal IH, Velioglu Y, Ener S: Femoral Nerve Palsy due to Anticoagulant Induced Retroperitoneal Hematoma. Case Rep Med 2014:450750

5. Kaiser MG, Haid RW, Jr., Subach BR, Miller JS, Smith CD, Rodts GE, Jr.: Comparison of the mini-open versus laparoscopic approach for anterior lumbar interbody fusion: a retrospective review. Neurosurgery 51:97-103; discussion 103-105, 2002

6. Mayer HM: A new microsurgical technique for minimally invasive anterior lumbar interbody fusion. Spine (Phila Pa 1976) 22: 691-699; discussion 700, 1997

7. Mummaneni PV, Haid RW, Rodts GE: Lumbar interbody fusion: state-of-the-art technical advances. Invited submission from the Joint Section Meeting on Disorders of the Spine and Peripheral Nerves, March 2004. J Neurosurg Spine 1:24-30, 2004

8. Murai Y, Adachi K, Yoshida Y, Takei M, Teramoto A: Retroperitoneal hematoma as a serious complication of endovascular aneurysmal coiling. J Korean Neurosurg Soc 48:88-90

9. Shen FH, Samartzis D, Khanna AJ, Anderson DG: Minimally invasive techniques for lumbar interbody fusions. Orthop Clin North Am 38:373-386; abstract vi, 2007

10. Silvestre C, Mac-Thiong JM, Hilmi R, Roussouly P: Complications and Morbidities of Mini-open Anterior Retroperitoneal Lumbar Interbody Fusion: Oblique Lumbar Interbody Fusion in 179 Patients. Asian Spine J 6:89-97

11. Sunga KL, Bellolio MF, Gilmore RM, Cabrera D: Spontaneous retroperitoneal hematoma: etiology, characteristics, management, and outcome. J Emerg Med 43:e157-161

12. Wang F: The diagnosis and treatment of traumatic retroperitoneal hematoma. Pak J Med Sci 29:573-576 